\title{
100 AÑOS DE EDUCACIÓN, PASTORAL Y SUEÑOS. BALTASAR PARDAL Y LAS HIJAS DE LA NATIVIDAD DE MARÍA
}

\author{
MARÍA TERESA IGLESIAS POLO ${ }^{1}$
}

Fecha de recepción: marzo de 2019

Fecha de aceptación y versión definitiva: septiembre de 2019

\begin{abstract}
Resumen: En 1918 nace en La Coruña un proyecto denominado La Grande Obra de Atocha, proyecto soñado y diseñado por Baltasar Pardal para dar educación gratuita y promoción social. Hoy son las Hijas de la Natividad de María las que lo mantienen vivo y las que han hecho que esta obra se adapte a los tiempos sin perder la esencia de los inicios.

Palabras clave: Baltasar Pardal; Grande obra de Atocha; Hijas de la Natividad de María.
\end{abstract}

\section{0 years of education, pastoral work and dreams. Baltasar Pardal and the Daughters of the Nativity of Mary}

ABSTRACT: In 1918 a project called the Great work of Atocha was founded in La Coruña. This project was envisioned and designed by Baltasar Pardal, aimed to provide free education and social advocacy. Today it is the Daughters of the Nativity of Mary who keep it alive and have adapted its work to the current times without losing the essence of its beginnings.

KEY WORDS: Baltasar Pardal; Great work of Atocha; Daughters of the Nativity of Mary.

1 CPR La Grande Obra de Atocha. Correo electrónico: mtiglesiaspolo@gmail. com. 


\section{INTRODUCCIÓN}

En 1918, Baltasar Pardal comienza un ambicioso proyecto de educación y promoción social en uno de los barrios más necesitados de La Coruña, que con el tiempo se extiende a otras ciudades y pueblos tanto de España como de Sudamérica. Además de crear esta obra, también es el fundador del instituto secular de las Hijas de la Natividad de María, que hoy en día siguen y mantienen esta obra, La Grande Obra de Atocha. Se define a Baltasar Pardal por las cuatro facetas que le acompañan toda su vida y que son sacerdote, catequista, pedagogo y fundador; en este trabajo nos centramos en lo educativo. Como se pone de manifiesto en estudios previos, Pardal no era pedagogo, pero no nos interesa tanto eso como que supo adaptar modelos pedagógicos a su escuela para favorecer el aprendizaje de los alumnos ofreciendo una enseñanza totalmente gratuita: «objetivo y magnitud de la obra. Ofrecer un local amplio, higiénico y cómodamente apropiado para dar enseñanza gratuita»(Plano del Salón Escuela Nota explicativa, 1923, p.1).

Antes de tener aprobada la autorización de funcionamiento del centro en 1923 tenía un proyecto sólido en el que se plasmaba cual sería el funcionamiento general y los principios que tendía de cara a la enseñanza, entre los que destaca su simpatía por el movimiento de la escuela nueva: «... Adosado al frente sur del gran salón y sin rebasar la altura del antepecho de los ventanales hay un cobertizo de cuatro metros de ancho por cuarenta y cuatro de longitud y ciento setenta y seis metros cuadrados para la enseñanza al aire libre» (Plano del Salón Escuela Nota explicativa, 1923, p. 2).

La escuela de Baltasar Pardal tiene como agente principal a la persona, bien sea un niño o un adulto, ya que a todos pretende llegar con la posterior inauguración de la Universidad Popular Femenina. Esta última dedicada a la formación de la mujer tanto a nivel instrucción elemental como de formación para el futuro con el objetivo de ayudar a su promoción social.

En un documento sin fechar, pero que por su clasificación en el archivo estaría entre 1918 y 1923, Pardal deja escrito el reglamento de lo que será la escuela que está fundando. Es interesante destacar en este escrito la distinción que hace entre escuela y Grande obra de Atocha como cosas unidas pero diferentes, y, como la primera depende directamente de la segunda, cuando define su finalidad: «El fin de la Escuela, como el de La Grande Obra de Atocha, es la formación de Generaciones Eucarísticas» (Pardal, 1923, p. 1).

Es esta escuela, y no la Grande Obra de Atocha, lo que vamos a analizar para poder entender los posteriores modos de hacer y el desarrollo del modelo pedagógico de las Hijas de la Natividad de María. 


\section{LA ESCUELA DE LA GRANDE OBRA DE ATOCHA}

La organización es algo presente en todos los proyectos de Pardal, desde que se ocupaba de la catequesis en la Capilla de Atocha y pasando por la inauguración de la Biblioteca del catecismo en 1914 ha dejado siempre por escrito las pautas de actuación y gobierno. El lema que tiene para la escuela, que tiene que ver con la finalidad de esta es «educar y salvar por medio de la Eucaristía» (Pardal, s.f., p.1). Hay que tener en cuenta, tanto en el fin como en el lema, que es un centro católico y también la importancia de la religión en los centros educativos de principios del siglo XX; este hecho no hace que hoy esté obsoleto en los centros dependientes de las Hijas de la Natividad de María, pero si se ha enriquecido con el objetivo de dar respuesta a la sociedad de cada momento.

En los procedimientos se pueden ver las influencias de Manjón y la escuela nueva, así como las también simpatías por la escuela activa. Además, en el mismo documento deja constatado que el fin principal de la Grande Obra de Atocha es la mujer y por ello dedicará más recursos a ella en la escuela. Con esto no deja de atender a los varones que de la misma manera les ofrece enseñanza sin depender de la edad o de los años de asistencia a las aulas, pero es a ellas a las que ofrece formación de adultas por las circunstancias del momento y las necesidades del barrio.

a) Haciendo y enseñando a hacer, a imitación del divino Maestro.

b) Instrucción y formación, tomando el catecismo como asignatura céntrica, ya que a Dios se le encuentra en todas las cosas y de Él hablan los cielos y la tierra.

c) Se utilizarán todos los métodos pedagógicos que mejor se adapten y más conducentes sean para los fines propuestos, teniendo en cuenta el carácter, circunstancias, etc.

d) La enseñanza será principalmente intuitiva y en cuanto sea posible al aire libre; enseñar jugando, atendiendo al desarrollo mental y corporal.

e) Como «La Grande Obra» será el «Hogar de los hogares» y al niño hay que educarle desde que aparece en el hogar, en las Escuelas de «La Grande Obra» no se señalaba edad para admitir a los niños.

f) Tampoco se señala edad para salir, especialmente para las niñas, ya que el fin específico de "La Grande Obra» es la formación de la mujer y a ella se la atenderá de un modo especial y formación postescolar.

g) La enseñanza será graduada para niños y niñas.

(Pardal, s.f, pp. 2-3) 
Habla Pardal de las escuelas como hogares, posiblemente sea de las primeras veces que utiliza este término para denominar a los centros como Escuela Hogar. Término que se ha mantenido hasta hace relativamente poco en los colegios de Pontedeume, Betanzos y Arzúa y que está evidenciado y justificado dentro del proyecto educativo institucional de las Hijas de la Natividad de María.

Otro aspecto importante en la escuela es el gobierno y en cuanto a este, los aspectos que destaca son los siguientes:

1) Los maestros serán de exclusivo nombramiento del Fundador de La Grande Obra o del Patrono de Patronos que se sucedan según las normas establecidas por el mismo.

2) A los maestros se les atenderá según los recursos de la Grande Obra, teniendo en cuenta que el interés principal ha de ser buscar primero el reino de Dios y su justicia y lo demás vendrá por añadidura.

3) Será director de las Escuelas el que lo es de La Grande Obra a quien corresponden la interpretación, modificación o ampliación del presente reglamento.

4) La enseñanza será completamente gratuita.

(Pardal, s.f., pp. 4-5)

De nuevo puede apreciarse la distinción entre La Grande Obra de Atocha y la escuela, lo que deja entrever que ya en ese momento tiene proyectos más amplios y variados dentro de la Institución que la educación. Otro aspecto interesante de resaltar es la visión de futuro, dejando así abierta la modificación o ampliación de este reglamento a futuras circunstancias.

Termina el documento con una conclusión breve en la que hace un resumen de lo que es el proyecto, poniendo de manifiesto que se fundamenta en la persona y en Dios, y concluyendo con una cita de Andrés Manjón y de las bases de la educación por la que apuesta.

Este no es más que un esquema del reglamento, pues el principal de la Grande Obra es la Caridad, que es vida de Dios y de los hombres, base del Hogar y de la Escuela, alegría para el mundo y luz para el maestro.

En esto consistirá toda la vida y espíritu de las escuelas de La Grande Obra cuyo ideal será enseñar al aire libre, enseñar jugando, enseñar haciendo, enseñar en humano, libre, español y cristiano, enseñar gratis a todo el mundo y enseñar paternal y socialmente cooperando con los demás educadores (P. Manjón).

(Pardal, s.f)

Con estos datos se puede evidenciar en la escuela de Baltasar Pardal y en el proyecto educativo de las Hijas de la Natividad de María una continuidad y una realidad que adaptada a cada momento tiene un fundamento en los planes iniciales del fundador. 


\section{MANERAS DE HACER EN LA ESCUELA DE LA GRANDE OBRA DE ATOCHA}

En este apartado vamos a realizar un análisis de las maneras de hacer en la escuela como respuesta a los principios plasmados en el reglamento, de manera que se pueda apreciar en el día a día cómo son tenidos en cuenta dentro del centro.

Aplicaba el método inductivo; suscitaba la creatividad personal de los alumnos, por ejemplo, pidiendo que dos niñas después de la misa, una dijese el evangelio y otra explicase; no descuidó ningún medio pedagógico de aprendizaje: láminas, representaciones, estandartes, exposiciones... entre las cuales destacaban las excursiones catequísticas.

(Agustinovich, 2006)

\subsection{ENSEÑAR AL AIRE LIBRE}

Pardal quiere un sistema de enseñanza en el que el niño sea el protagonista y pueda aprender y crecer en relación con la comunidad y la naturaleza. Este es el motivo por el que, en los planos de La Grande Obra de Atocha, aparecen espacios destinados a la docencia fuera de las aulas y se refleja en la importancia de cobertizos para poder estar en los días de lluvia, las salidas y excursiones que realiza con el alumnado. Cuando visitó las escuelas del Ave María en Granada fue por esto por una de las cosas por las que más se interesó; no quiere niños sentados memorizando contenidos, quiere que estos aprendan de una manera más activa y participativa.

\subsection{ENSEÑAR JUGANDO}

La enseñanza lúdica es algo importante en la escuela; jugando, los contenidos se asimilan más rápidamente y permanecen durante más tiempo en la memoria de los alumnos. En este sentido son múltiples y variados los recursos que Pardal utiliza y que adapta al nivel de los alumnos de la misma manera que indica el fundador de las escuelas del Ave maría cuando dice que «el juego es la única asignatura de los niños hasta los cinco años; la principal de los seis a los nueve; la indispensable, de los diez a los catorce; y la más saludable e higiénica hasta los veintiún años; y el educador que de ella no se ocupe ni preocupe, no sabe ni vale para educar» (Manjón, 1949, 
p. 351). Se utilizan en la escuela canciones para aprender contenidos de las diferentes asignaturas de manera que cantando les resulte más sencillo adquirir el conocimiento.

La metodología que se utiliza está basada en el juego, siempre que se puede, se enseña de manera lúdica, algunos ejemplos cogidos de Manjón son la Rayuela para aprender historia, o el mapa de España para aprender geografía, que sitúa en el patio de recreo.

ILUSTRACIÓN 1.

RAYUELA Y MAPA DE ESPAÑA EN UN COBERTIZO DE LAS ESCUELAS DEL AVE MARÍA, GRANADA

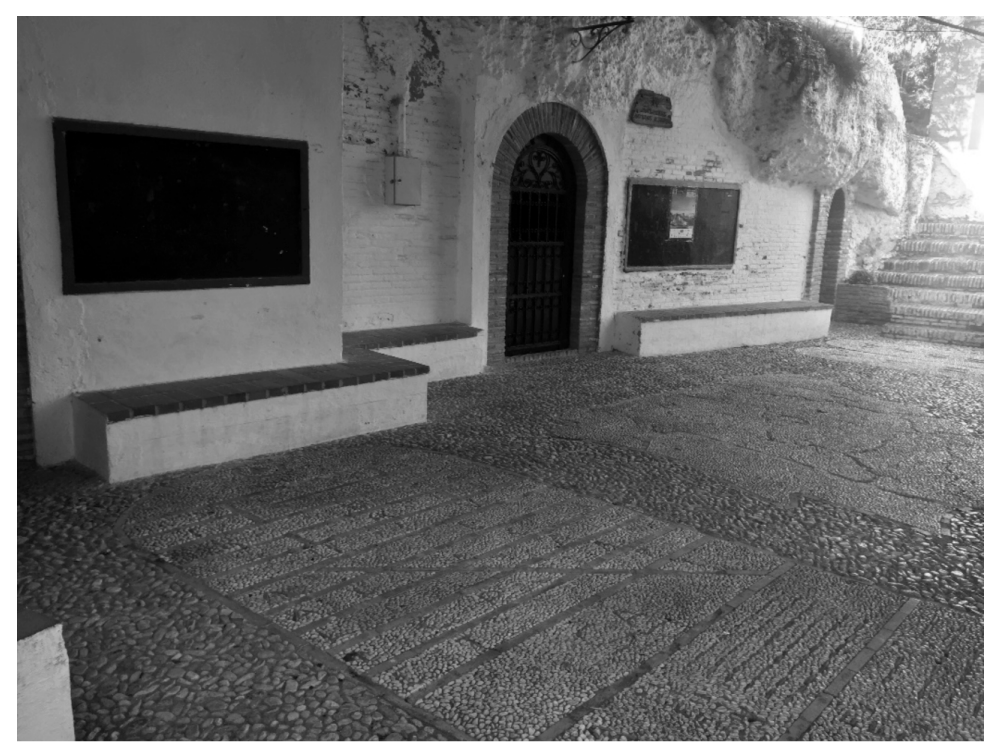


ILUSTRACIÓN 2.

\section{RAYUELA Y MAPA DE ESPAÑA EN UN COBERTIZO DE LA GRANDE OBRA DE ATOCHA-A CORUÑA}

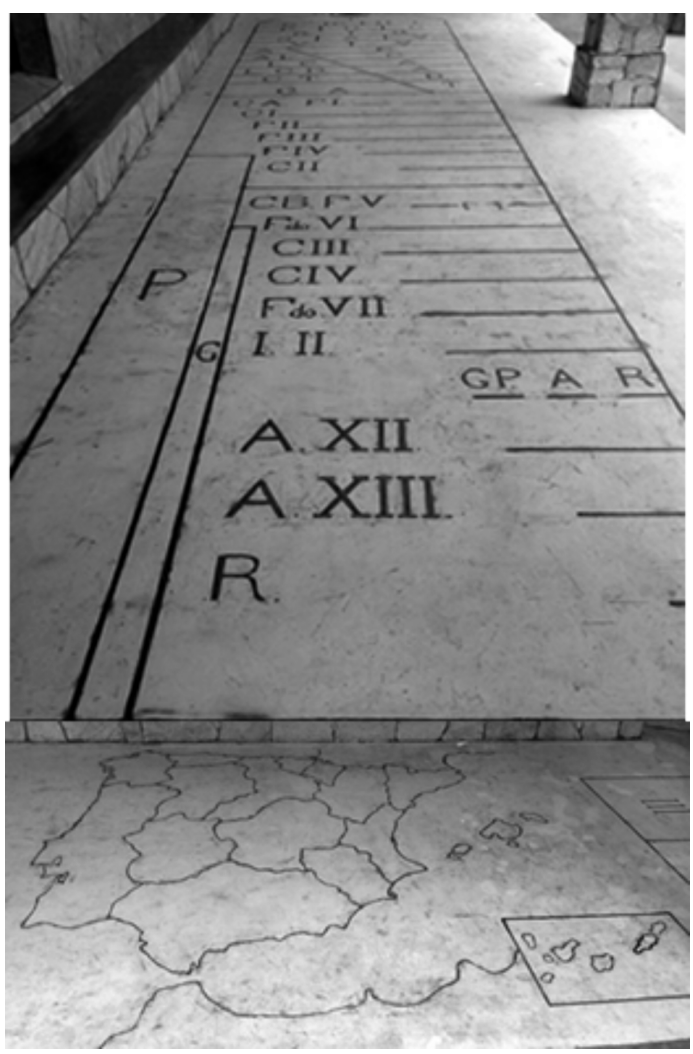

La rayuela que tiene como finalidad enseñar historia de España, sigue un procedimiento para su correcto funcionamiento que encontramos en Manjón (1948, p. 261) y que destaca que en estas clases lo importante no es conseguir que el niño adquiera muchos datos concretos, sino pocos, pero los más importantes o característicos de cada momento.

El mapa de España, para la geografía tiene también unas pautas de funcionamiento que Manjón indica que «procuro ir de lo conocido y visto a lo por conocer, y empiezo por la escuela y lugar de ella, haciendo un croquis, que voy ampliando, pasando a la provincia, región, etc.,» (1948, p. 261). Además, para geografía se emplean también proyectos a modo de juego que implican a todos los niños como agentes activos y partícipes. 
Pero lo que más me interesó de cuanto llevaba visto fue la magna obra que un grupo de escolares realizaba al empedrar una plazuela, reproduciendo los contornos de un gran mapa de España. Los más pequeños escogían las piedras y las clasificaban según sus formas, colores y tamaños; otros trazaban las sinuosidades del litoral de Cataluña, copiando con la fidelidad posible la silueta, pintada en un cartón, que les servía de modelo; varios iban rellenando con piedras diferentes la tierra y el mar en las regiones cuyo trazado había sido aprobado ya sin duda por los directores de la obra, y todos alternaban en los trabajos, discutían su exactitud e ilustraban las cuestiones con las noticias y juicios personales que tenían o formaban sobre ellas. Nadie estaba inactivo; la Geografía entera de España andaba de boca en boca, un tanto corrompida a veces y con algún que otro error nada pequeño, pero siempre corregida en forma y fondo por alguno de aquellos Aristarcos en agraz, pues jamás perdonaban los errores ajenos que estuvieran al alcance de su propia ciencia. Y como por menguada que fuera la de cada uno, era estimable la que reunían entre todos, resultaba de aquella confusión aparente una instrucción mutua tan eficaz y positiva, que de seguro al concluir la obra, que era por cierto de bastantes días, a juzgar por lo poco que adelantó a mi vista, saldría sabiendo cada uno por lo menos tanto como al empezar supieran entre todos.

Y no será sólo esto: allí ejercitaban la observación, la comparación de proporciones, la estimación de las distancias y hasta el razonamiento ${ }^{2}$.

\subsection{ENSEÑAR HACIENDO}

En el punto anterior ya puede apreciarse un ejemplo de la manera de enseñar haciendo; pero encontramos más con el carácter festivo que se integraba en la pedagogía de la escuela y que se aprovechaba la preparación de fiestas o representaciones para integrar contenidos curriculares. Con la preparación de las escenificaciones se consigue que el niño sea protagonista tanto de las representaciones como de la construcción de su propio aprendizaje.

\subsection{ENSEÑAR EN HUMANO, ENSEÑAR LIBRE}

El agente principal de la grande obra es el ser humano, independientemente de sus características o circunstancias. Así la escuela de la Grande

2 Relato de las impresiones de la visita a las escuelas del Ave maría por D. Federico Olóriz en el Ateneo de Madrid en sesión dedicada a propagar las Fundaciones Manjón. 16 de diciembre de 1989. En Galvez Carmona, G. Pedagogía Española, p. 319. 
Obra de Atocha pretende llegar a todos partiendo de sus diferencias no de la homogeneidad de los grupos. El principio de la inclusión y la integración está en fortalecer en cada individuo aquello en lo que demuestra carencias; es por esto importante ver la pluralidad y enriquecerse con ella puesto que esta es la persona creada por Dios, con semejanzas y diferencias. El hombre, aunque con espíritu transcendente es hombre y tiene necesidades que pueden impedir el desarrollo de otras facultades, de esta manera primero mira a la persona y luego a lo que puede llegar a ser en potencia. Algunos ejemplos de esta humanidad se encuentran como máximas del fundador cuando afirma que «una ver fortalecida la vida física, ya puede el educando cultivar y perfeccionar todas sus facultades en la Grande e Higiénica escuela en donde los sentidos aspiren el despejado ambiente, mientras el alma recibe el beneficio inestimable de la educación» (Pardal, 1919, [2] p.4).

\subsection{ENSEÑAR ESPAÑOL Y CRISTIANO}

En el momento que Pardal comienza a planificar la escuela, el sentimiento de España como patria es algo muy importante en la educación y además de la geografía también se trabaja para que los niños se sientan agentes de una nación y una historia común. En este sentido se utilizan en la escuela recursos como canciones o láminas, de manera que los niños puedan aprender por medio de recursos visuales y auditivos.

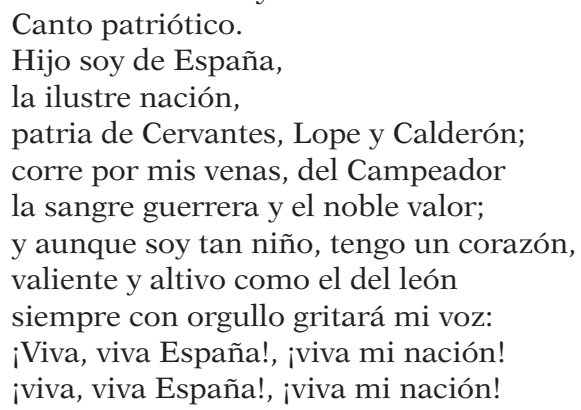

(Pardal, sf)

Se usan estampas de cuadros de Murillo con representaciones de la vida de la Virgen y de Jesús para trabajar la catequesis. En las paredes se encuentran treinta y seis máximas y en los distintos espacios aparecen láminas que ayudan a las explicaciones del evangelio y la religión. 
En el piso primero de este pabellón, en láminas artísticamente colocadas a lo largo de las paredes, se representa el pensamiento católico distribuido en tres salas.

a) El papado. En la sala central está representada la jerarquía eclesiástica con el busto en lámina de todos los papas.

b) La eucaristía y vida de la Iglesia. Al lado de esta sala central, tiene por titular a San Tarsicio, el niño mártir de la Eucaristía.

c) Expansión de la Iglesia, por obra de las misiones. Termina esta representación en la sala de Santa Teresita del Niño Jesús con gráficos de la Organización misionera, vistas y escenas de las Misiones.

En el segundo piso de este pabellón se desarrolla también en láminas la idea patriótica.

d) Galicia. En la primera sala dedicada a San Pelayo, niño santo y gallego, aparecen las vistas de los monumentos principales de Galicia empezando por la parte principal Santiago de Compostela.

e) España monumental. Ocupa el centro de esta Sección la sala de San Fernando, Rey de España, con vistas de todas las provincias.

f) América. Finalmente, en la tercera sala de la Virgen del Pilar, se representa el pensamiento de la expansión española, que empieza con el descubrimiento de América, expuestos en grandes cuadros los planes y viajes de Cristóbal Colón, con reproducción fotográfica de la carabela «San María» y El Quijote resumido en láminas como símbolo del lenguaje.

g) Isabel la católica. Completa este conjunto simbólico el cuadro con el testamento de Isabel la Católica. Y preside la sala la Virgen del Pilar para representar la formación de la mujer desde niña.

(Pardal, 1950, pp. 17-18)

\subsection{ENSEÑAR GRATIS A TODO EL MUNDO}

La educación de la escuela de la Grande Obra de Atocha, es para todo el mundo sin tener edad de comienzo ni de finalización; de ahí que lo visual fuera un punto importante ya que puede llegar a todos independientemente de la edad. Teniendo en cuenta el carácter pastoral de la institución muchos de los esfuerzos se centraron en llevar el Evangelio a la población y es por eso que gran cantidad de recursos estuvieran adaptados a este fin: «El 8 de septiembre de 1922, al terminar la novena de la Virgen de Atocha, tiene D. Baltasar la genial y luminosa idea de comprometer a Nuestro Señor, para 
poder celebrar al año siguiente la novena en el salón de la Grande Obra. Este compromiso puso en movimiento a todos. Desde aquel momento empezó una actividad grande, no sólo en La Coruña, sino en muchas partes de España a donde se mandaron telas de raso de varios colores para confeccionar más de 170 estandartes relativos a los Anuncios y Profecías de Jesús y María contenidos en la Biblia y se hicieron otros tantos encargos a este fin» (Tenreiro, s.f. p. 25).

Estos estandartes son pinturas originales con representaciones eucarísticas, marianas y otras varias que sirven como apoyo en la catequesis y en la transmisión del mensaje de Dios y de la historia de la Iglesia. Son cincuenta y nueve los que se dedican a la Eucaristía mostrando pasajes del antiguo testamento; a María se le dedican 89 y hacen un recorrido desde la mujer en el antiguo testamento hasta María en el Evangelio. Los restantes son de temas diversos.
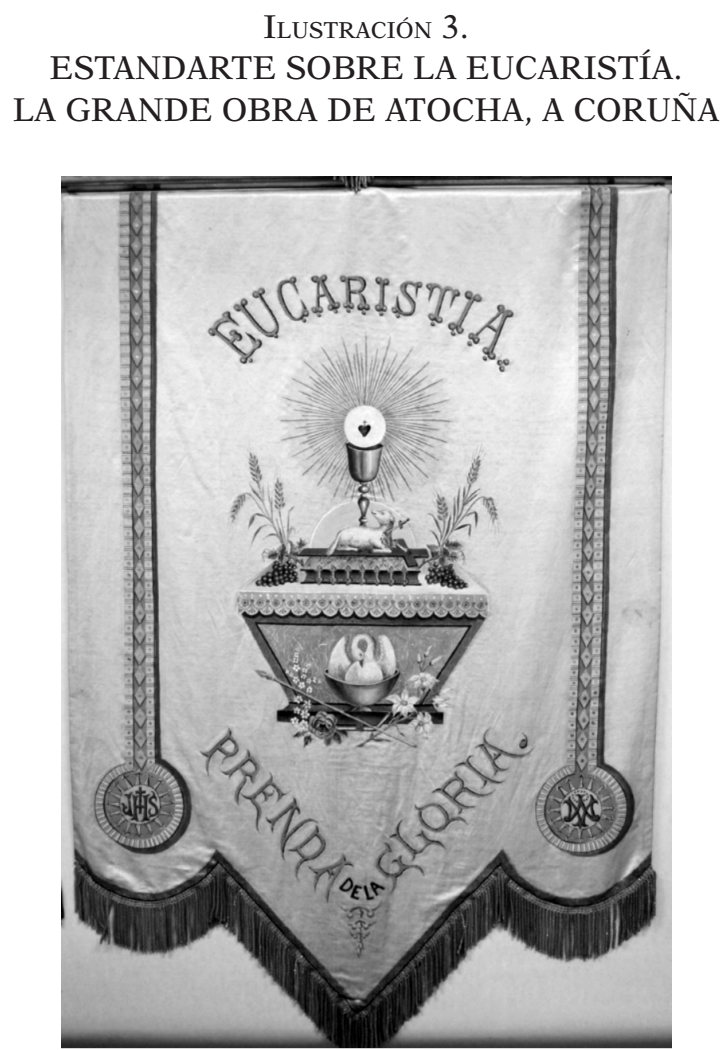
ILUSTRACIÓN 4.

ESTANDARTE SOBRE MARÍA. LA GRANDE OBRA DE ATOCHA, A CORUÑA

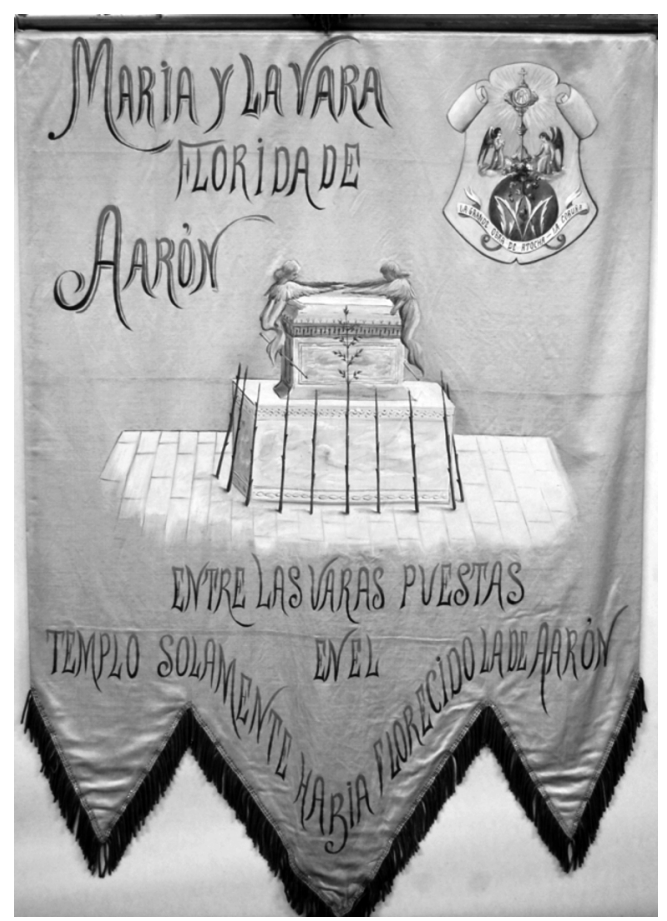

3.7. ENSEÑAR PATERNAL Y SOCIALMENTE COOPERANDO CON LOS DEMÁS EDUCADORES

Aboga el fundador porque la escuela de La Grande Obra de Atocha, sea como un hogar para los alumnos y es en la familia en la que fundamenta la educación eficaz. Es sobre todo la madre la responsable de la formación de los hijos a nivel ético y moral puesto que con las largas jornadas laborales de principios de siglo muchos de los padres apenas tienen tiempo para ver a los niños. Las madres en la mayoría de los casos no tienen capacidad para enseñar letras o número, ya que el analfabetismo femenino en los años veinte del siglo pasado ronda el 90\%, pero si para transmitirles valores que hagan de ellos honrados ciudadanos. Por este motivo, Pardal (1916) la pone 
como agente con mayor fuerza en esta labor cuando dice que «Educar a la mujer es educar hombre y levantar pueblos» y que la Obra de Atocha «tiende principalmente a la formación de la mujer, como base de la familia y de los hogares eucarísticos».

De la misma manera que considera esencial educar con amor de familia también destaca la importancia de la colaboración entre todos los implicados en el proceso de formación. Educar es tarea de toda la sociedad y dentro de la escuela de todas las personas que la componen caminando juntos es busca del mismo fin. Para que este modo de actuar sea una realidad organiza reuniones periódicas en las que se valora el trabajo realizado y se proyectan nuevos retos en función de las necesidades y resultados obtenidos previamente.

\section{LA ESCUELA DE LAS HIJAS DE LA NATIVIDAD DE MARÍA}

Con el objetivo de mantener vivo el espíritu del fundador de la Grande Obra de Atocha, Baltasar Pardal, a la vez que fortalecer la unidad, desde las Hijas de la Natividad de María se diseña un proyecto común a todas las obras. El propósito de este es garantizar la consecución permanente de la identidad de manera que todas las personas implicadas tengan una guía de actuación.

Nuestra misión es continuar y propagar la Obra de nuestro Fundador, quien creó esta Institución católica de carácter benéfico-docente (1923) para paliar y satisfacer las necesidades materiales y morales de un barrio marginal coruñés (barrio de Atocha) y elevarlo a un nivel de Dignidad Humana y Ética.

(PEI, 2010, pp. 1-2)

En este documento quedan reflejadas las bases fundamentales del proyecto educativo por las que apuesta la institución que tienen un carácter tanto educativo como de apostolado. Este trabajo se centra en la parte educativa de la Institución que por el carácter católico que tiene no pierde de vista la pertenencia a la unidad de la Iglesia.

Se busca la manera de formar alumnos competentes y comprometidos; competentes en la vida y comprometidos en la sociedad. Este hecho motiva que se tenga en cuenta en los centros la necesidad de programar ambiciosos proyectos que den a los alumnos tanto formación académica, como estrategias y herramientas para ser resolutivos más allá de los años escolares. 
Pretendemos [...] que los alumnos/as cuando salgan de nuestros centros sean personas libres, creativas, flexibles, autónomas, comprometidas socialmente, que tengan un proyecto de vida, un compromiso cristiano, e intelectualmente competentes.

(PEI, 2010, p. 3)

En la sociedad actual, los avances tecnológicos hacen que tanto la ciencia como la información cambien constantemente; por este motivo es necesario no perder de vista esta evolución constante, ya que hay una demanda patente de nuevas metodologías, nuevas formaciones, etc., Avances como el acceso rápido a la información desde cualquier lugar y en cualquier momento hacen necesaria una capacitación en la gestión de la misma, hay que dar respuestas a nuevos retos y por ello la formación permanente de calidad es algo esencial para lograr el éxito esperado en la formación de los alumnos de los centros.

Escuelas en constante reflexión sobre la acción educativa comprometidas en la consecución de nuevas metas-objetivos, afrontando los cambios como retos ilusionantes para conseguir alumnos/as felices, y personal, social y espiritualmente competentes.

(PEI, 2010, p. 3)

\section{CONCLUSIONES}

En el Proyecto educativo Institucional podemos encontrar los rasgos significativos de la pedagogía de las Hijas de La Natividad de María, fundamentada en los principios del fundador que es quien marcó las primeras líneas de actuación. En cada momento histórico las necesidades son diferentes y por consiguiente las demandas también, así desde los orígenes se ha ido actualizando el proyecto para poder ofrecer lo que, en cada momento, la sociedad requiere, pero siempre teniendo presentes los rasgos marcados en los inicios de la Institución que marcan el compendio de su pedagogía que es «enseñar haciendo, para educar enseñando»(PEI, s.p.). Aparecen también recogidas en este documento las bases en las que se fundamenta el ideario pedagógico de las Hijas de la Natividad de María haciendo referencia a la tradición mantenida desde los inicios y que se hace patente en los centros. 


\section{REFERENCIAS}

Agustinovich, A. (2006). Un cura, evangelio viviente, Don Baltasar Pardal. Santiago de Compostela: Cátedra Baltasar Pardal.

Gómez, M. D. C. G., Vidal, J. E., y Polo, M. T. I. (2017). Baltasar Pardal. Fundador de La Grande Obra de Atocha, una institución creada para educar a la mujer. Revista Brasileira de História da Educação, 17(4), 3.

Gómez, M. C., Escudero, J., e Iglesias M. T. (2016). Influencias pedagógicas de Andrés Manjón en Baltasar Pardal. Cabás: Revista del Centro de Recursos, Interpretación y Estudios en materia educativa (CRIEME) de la Consejería de Educación, Cultura y Deporte del Gobierno de Cantabria (España) [en línea], 15, 131-144.

Gómez, M. C. G., y Polo, M. T. I. (2017). La Grande Obra de Atocha, una aportación de la Iglesia a la sociedad. Revista Electrónica de Educación Religiosa, Didáctica y Formación de Profesores., 6(2).

Gómez, M. D. C. G., y Polo, M. T. I. (2017). Baltasar Pardal Vidal y la Grande Obra de Atocha. Padres y Maestros/Journal of Parents and Teachers, (370), 78-80.

Iglesias, M. T. (2015). D. Baltasar, de la nada al todo. A Coruña: Cátedra Baltasar Pardal.

Iglesias, M. (2011). Baltasar Pardal Vidal, educador y catequista. Santiago de Compostela: Cátedra Baltasar Pardal.

López, G. (2006). Don Baltasar, ¿un hombre corriente? La Coruña: Asociación Baltasar Pardal.

Manjón, A. (1948). El pensamiento del Ave maría. Patronato de las escuelas del Ave María.

Montero, J. (2002). Siguieron sus pasos... Poveda, Fenollera, González y Pardal, cuatro fundadores que siguieron las huellas de A. Manjón. Granada: Centro de Estudios Pedagógicos y Psicológicos Andrés Manjón.

Pardal Vidal, B. (1914). Fundación de la biblioteca de Nuestra Señora de Atocha. La Coruña.

Pardal Vidal. B. (1950). La Grande Obra de Atocha. Su origen, constitución y funcionamiento. La Coruña: Moret.

Pardal, B. (sf). Reglamento de las Escuelas de La Grande Obra de Atocha.

- (1919). La Grande Obra de Atocha, ¿qué es y qué pretende? Revista La Grande Obra de Atocha. (2).

- (1926). El libro del maestro: el niño. La Coruña: Imp. El Ideal Gallego.

- (1933). El AZ de a catequista, o lo que no debe ser en su sección. Revista catequística. (227), pp. 266-268.

- (1938). Valor pedagógico del evangelio. Misión. Suplemento 151.

Seoane. (2002). Comunicación personal con José Montero exdirector general de las escuelas del Ave María, 27 de abril de 2002.

Seoane Faraldo, T. (1996). Una vida de fe y confianza: Baltasar Pardal. La Coruña: Diputación Provincial.

Seoane Faraldo, T. (1996). Un gran catequista Don Baltasar. Santiago de Compostela: HNM. Imprenta Mundo.

Tenreiro Tenreiro, M. (1985). Una vida consagrada a la niñez y a la juventud. Pontedeume: E.H. San José. 